\title{
A unified theory of aerosol coagulation
}

\section{M R Williams}

Nuclear Engineering Department, The University of Michigan, Ann Arbor, Ml 48109, USA

Heceived 14 December 1987, in final form 9 February 1988

\begin{abstract}
A unified theory of the coagulation of aerosols is presented based upon the solution of a generalised diffusion equation which describes classical diffusion due to Brownian and turbulent motion as well as the effect of an overall relative velocity arising, for example, from gravitational settling and inertial turbulent motion. Certain statistical assumptions regarding the turbulent motion enable exact solutions of the diffusion equation to be obtained for the four mechanisms mentioned above. As a result, we can assess the accuracy of the usual procedure of adding the coagulation kernels. It is also possible to include the effects of inter-particle fluid forces which result in a collision efficiency less than unity. In contrast to previous work on this subject, the collision efficiency affects all processes and not just gravitational settling. Significant deviations from the classically computed coagulation kernels are noted.
\end{abstract}

\section{Introduction}

The distribution in size of an aerosol is determined by a number of physical phenomena. For example, the manner in which the aerosol particles diffuse to boundaries and settle out due to gravitational or electrical forces governs the removal rate. In addition, the relative motion of individual particles leads to a 'fusion' process which is termed coagulation. Such a mechanism can arise from many simultaneously acting processes. Thus, Brownian motion, gravitational sedimentation, turbulence and several other processes which can be induced by applied forces all contribute to the coagulation rate (Friedlander 1977). In order to obtain the net effect of these phenomena, it is necessary to solve the integro-differential balance equation for the particle volume distribution function $n(v, t)$. Such an equation can be written (Lushnikov 1974, Friedlander 1977):

$$
\begin{aligned}
& \frac{\partial n(v, t)}{\partial t}+R(v) n(v, t)=\frac{1}{2} \int_{0}^{v} \mathrm{~d} u K(u, v-u) \\
& \quad \times n(u, t) n(v-u, t)-n(v, t) \int_{0}^{\infty} \mathrm{d} u K(u, v) \\
& \quad \times n(u, t)+S(v, t)
\end{aligned}
$$

where $R(v)$ is the removal rate per unit time due to diffusion, gravitational settling and indirectly to coagulation. $K(u, v)$ is the coagulation kernel and $S(v, t)$ is an independent source term. Equation (1) assumes a well mixed background gas but can be modified to describe a more detailed spatial dependence of the aerosol distribution if necessary. For present purposes, equation (1) is sufficient since the object of this work is to describe the way in which the coagulation kernel can be calculated in more detail than has been customary in the past. In particular, we wish to direct attention to Brownian motion, gravitational sedimentation and turbulent mixing.

In general, these phemonena have been studied separately and coagulation kernels obtained for each mechanism. The resultant coagulation kernel is then assumed to be the sum of each mechanism. There is no a priori reason for this assumption and some work has been done to verify it. For example, Saffman and Turner (1956) have examined the simultaneous action of turbulent and gravitational coagulation and found that a root mean square average (Papoulis 1965) of the individual kernels is more appropriate than an arithmetic sum. There is a number of assumptions inherent in the work of Saffman and Turner; namely, a collision efficiency of unity, thereby restricting the results to nearly equal sized droplets, the assumption of a Gaussian distribution of relative velocities for the particles and neglect of the directional effect of gravitational coagulation. It is the assumption of a Gaussian distribution, for example, that leads to the root mean square average. Improvements to this work which account for the collision efficiency of non-equal droplets (Pertmer and Loyalka 1980) and the limitation of the Gaussian assumption need to be introduced. An analogous problem exists when Brownian and gravitational coagulation occur simultaneously. Simple calculations show that Brownian coagulation is the dominant 
mechanism for particles less than about 1 micrometre in diameter and, thereafter, because of increasing particle mass, gravitational coagulation takes over. The accepted approximation is to simply add the Brownian and gravitational kernels in the hope that when one is small the other is large. Recent calculations (Simons et al 1986), in which the combined problem has been solved exactly, show conclusively that significant errors can arise in the region where the ratio of inertial to diffusion effects is around 6 . There is, therefore, a need for a consistent approach to the calculation of coagulation kernels which are at the core of any assessment of aerosol removal.

In the present work we shall describe, by means of a generalised diffusion equation, a self-consistent method for calculating coagulation kernels from all mechanisms cited above. In some cases, analytical results can be obtained and relatively simple expressions deduced for $K(u, v)$. In more complex situations, numerical solutions will be required. At each stage, however, it is possible to estimate the error involved and understand the nature of the approximation made. The resulting coagulation kernels are then available for insertion into environmental and atmospheric problems and this is of considerable significance in the improvement of nuclear safety computer codes which are designed to predict the dispersal and deposition of radioactive aerosols (Silberberg 1979).

A final point to note is that little or no development work is being carried out in the area of coagulation kernels. Such work as is known to the author concerns the calculation of collision efficiencies for gravitational coagulation in isolation. That work is directed towards calculating particle trajectories arising from the perturbing fluid forces between interacting spheres (Pruppacher and Klett 1978, Hocking 1959). The collision efficiencies so obtained are employed as correction factors to the gravitational coagulation kernel, which in turn is included either in the additive or the root mean square assumptions. Our proposals use these calculations but in the more consistent framework of a generalised diffusion equation.

\section{Methodology}

\subsection{The diffusion equation}

Particle sizes of interest in aerosol problems range from 0.001 to about 100 micrometres. Thus they span a region in which the microscopic details of the fluid medium are significant, i.e. size of order of a mean free path up to the region where continum mechanics can be employed with great accuracy. In principle, therefore, any definitive study of such colliding particles should use the linear Boltzmann transport equation for the fluid effects with the presence of the particle introduced by a suitable boundary condition. For the calculation of drag forces on single particles (generally spherical) this technique has been developed to a high degree of precision by
Cercignani and others (Cercignani 1975) with the complete range of Knudsen numbers (mean free path/diameter) being covered. Extension to other shapes is not simple but could in principle be done numerically. When two bodies are considered, however, the problem of solving the Boltzmann equation becomes exceedingly laborious and as far as the author is aware has never been attacked; although it is not beyond the realm of possibility that a Monte Carlo or finite-element technique could be used.

Even if the Boltzmann equation were employed, however, it is not clear that the necessary physical situation could be modelled adequately. For example, it would be necessary to consider two coupled Boltzmann equations: one for the fluid and one for the particles. Small particles, less than a mean free path in size, could be regarded as a special species of gas molecule and treated accordingly. But larger particles would significantly modify the gas distribution function in their neighbourhood and this would introduce many difficulties. It would be necessary, for example, to include the bodies as boundary conditions: a difficult problem. Moreover it would still be necessary to incorporate Brownian diffusion and gravitational forces and, in addition, any turbulent forces prevailing. Such difficulties suggest that it is more profitable to abandon a detailed description of particle motion and gas atom motion which rely upon microscopic velocity distributions and look at a macroscopic description in which the microscopic properties appear in the form of diffusion coefficients, viscosity, etc.

This lower level of description is best carried out in terms of a generalised diffusion equation as described by Chandrasekhar (1943). We seek, therefore, an equation which will describe the particles simply by their concentration in the fluid, e.g. $C(\boldsymbol{r}, t)$, where $C(\boldsymbol{r}, t) \mathrm{d} \boldsymbol{r}$ is the number of aerosol particles in the volume element $\mathrm{d} r$ at $r$ at time $t$. Such a diffusion equation may be written

$$
\frac{\partial}{\partial t} C(\boldsymbol{r}, t)=\boldsymbol{\nabla} \cdot \boldsymbol{J}(\boldsymbol{r}, t)
$$

where $J$ is the current of particles of one type onto a test particle. The expression for the current can be written

$$
\boldsymbol{J}(\boldsymbol{r}, t)=-D(\boldsymbol{r}, t) \boldsymbol{\nabla} C(\boldsymbol{r}, t)+\boldsymbol{V}(\boldsymbol{r}, t) C(\boldsymbol{r}, t)
$$

where $D$ is the mutual diffusion coefficient describing the effect of essentially stochastic forces acting on the particles and $\boldsymbol{V}$ is a drift velocity arising, for example, from gravitational or fluid effects.

Since we shall be concerned with spherical particles (mainly because a consistent formalism for non-spherical particles does not exist), we shall consider the diffusion of particles of radius ' $b$ ' onto a test particle of radius ' $a$ '. We will also assume that a steady-state situation prevails and hence the diffusion equation becomes

$$
\boldsymbol{\nabla} \cdot D(\boldsymbol{r}) \boldsymbol{\nabla} C(\boldsymbol{r})-\boldsymbol{\nabla} \cdot(\boldsymbol{V}(\boldsymbol{r}) C(\boldsymbol{r}))=0 .
$$


In spherical polar coordinates, with the origin at the centre of the ' $a$ ' particle, we have

$$
\begin{aligned}
\frac{1}{r^{2}} \frac{\partial}{\partial r} & \left(r^{2} D(r) \frac{\partial C}{\partial r}\right)+\frac{D(r)}{r^{2} \sin \theta} \frac{\partial}{\partial \theta} \\
& \times\left(\sin \theta \frac{\partial C}{\partial \theta}\right)+\frac{D(r)}{r^{2} \sin ^{2} \theta} \frac{\partial^{2} C}{\partial \varphi^{2}} \\
& -\frac{1}{r^{2}} \frac{\partial}{\partial r}\left(r^{2} V_{r} C\right)-\frac{1}{r \sin \theta} \frac{\partial}{\partial \theta} \\
& \times\left(\sin \theta V_{\theta} C\right)-\frac{1}{r \sin \theta} \frac{\partial}{\partial \varphi}\left(V_{\varphi} C\right)=0
\end{aligned}
$$

where $D(\boldsymbol{r})$ is assumed to depend only on the radial coordinate in view of the microscopic isotropy of the fluid. $V_{r}, V_{\theta}, V_{\varphi}$ and $C$ are general functions of the independent variables $(r, \theta, \varphi)$.

If the velocity $V$ satisfies $\boldsymbol{\nabla} \cdot \boldsymbol{V}=0$ as in the case of an incompressible fluid, the equation simplifies considerably, but this is not necessarily the case for solid particles.

The boundary conditions that have to be imposed on the diffusion equation are as follows:

$$
\lim _{r \rightarrow \infty} C(r, \theta, \varphi)=C_{x}
$$

and

$$
C(a+b, \theta, \varphi)=0 .
$$

The latter condition implies that the spheres are impenetrable and that they coalesce on touching. The quantity of interest as far as aerosol coagulation is concerned is the number of particles captured per unit time namely:

$$
N=-\int_{s} J \cdot \mathrm{d} S
$$

where the integral is to be taken over the sphere $r=$ $a+b$. Since on this sphere $C=0$, we find

$$
N=D(a+b) \int_{s} \nabla C \cdot \mathrm{d} S
$$

which in polar coordinates become

$$
\begin{aligned}
N= & (a+b)^{2} D(a+b) \int_{0}^{2 \pi} \mathrm{d} \varphi \\
& \times\left.\int_{0}^{\pi} \mathrm{d} \theta \sin \theta \frac{\partial C}{\partial r}\right|_{r=a+b}
\end{aligned}
$$

thus the total number of coagulations of ' $a$ ' and ' $b$ '-type particles is $n N$ where $n$ is the number density of ' $a$ '-type particles and the coagulation kernel is by definition

$$
\begin{gathered}
K(a, b)=\frac{(a+b)^{2}}{C_{\star}} D(a+b) \int_{0}^{2 \pi} \mathrm{d} \varphi \\
\times\left.\int_{0}^{\pi} \mathrm{d} \theta \sin \theta \frac{\partial C}{\partial r}\right|_{r=a+b} .
\end{gathered}
$$

The problem reduces therefore to the solution of the generalised diffusion equation (5). In formulating the problem in this way, we have exchanged one set of problems for another in that it is now necessary to calculate $D(r)$ and $\boldsymbol{V}(r)$.

\subsection{The diffusion coefficient}

In classical diffusion theory, the diffusion coefficient refers to an isolated particle in an infinite fluid. When diffusion of one particle to another is involved, the assumption is made, on the basis of a Gaussian distribution in position, that the mutual diffusion coefficient is the sum of the two individual values. This assumption neglects the interaction of the two particles with each other via the fluid and hence the distortion in the fluid velocity. When these effects are included, the mutual diffusion coefficient becomes a function of particle separation. For particles which are well separated, the classical result is valid but when they approach closely the effective diffusion coefficient decreases markedly (Spielman 1970, Batchelor 1976). Such effects have recently been included in the calculation of coagulation in the case of pure Brownian interaction (Shahub and Williams 1988).

There is a further dependence of $D$ on position arising from turbulent diffusion. From the eddy viscosity concept and the theory of isotropic turbulence, it is known that, to a good approximation, a turbulent diffusion coefficient can be employed which, for eddies that are smaller than the turbulent microscale, is proportional to the square of the distance between the particle centres. Such concepts have been employed with some success in the calculation of turbulent deposition (Crump and Seinfeld 1981, Levich 1962). Thus the functional dependence of $D$ on position is available.

\subsection{The drift velocity}

An overall relative drift velocity between particles can arise from a variety of causes. For example, unequal particles fall at different speeds under the action of gravity. They also move with different relative velocities under the action of turbulence when particles are not fully entrained in the eddies: thus the particle's random inertia leads to a form of turbulent coagulation which depends on mass difference. Such an effect is in addition to the turbulent diffusion effect mentioned above. Expressions for the turbulent velocity arising from this phenomenon can be obtained in terms of the intensity of turbulence (Batchelor 1951). Another mechanism leading to a drift velocity arises from an electric field acting on a charged aerosol particle. This effect introduces a force equal to $q E$ where $q$ is the charge on the particle and $E$ is the applied electric field. Induced charge may also be important in this case. However, even with no applied field, a charged particle generates an external field and this will influence other particles in its neighbourhood. The radial velocity so generated will be related to the frictional drag on the particles and their charges.

A final point that should be noted in the calculation 
of drift velocity is the effect of the collision efficiency. Due to the mutual interaction of the particles (in the same way as occurs in mutual diffusion) there is a distortion of the velocity field which affects the trajectories of the particles relative to one another. Thus two particles which would collide on purely geometric arguments, will often not collide because fluid forces cause them to be deflected. The calculation of such effects has been studied for many years (Pertmer and Loyalka 1980, Hocking 1959) and it is relatively straightforward to incorporate these modifications to the flow into the formalism described above.

\subsection{Kinetic theory effects}

When the size of particles becomes comparable to a mean free path of the gas atoms in the surrounding fluid, many of the continuum theory methods fail. To develop a detailed microscopic approach based on the Boltzmann equation is not practicable nor is it necessary for most practical aerosol problems. A variety of methods exist for incorporating such kinetic theory effects into continuum theory formalisms. For example, the Stokes-Cunningham correction is a semi-empirical factor by which diffusion coefficients can be multiplied and which extends their validity well into the Knudsen regime. Another method, which is particularly suited to the proposed approach, is to use the Fuch's jump technique (Hidy and Brock 1970). In this approach, it is assumed that a fictitious spherical shell exists around the particle of interest. The thickness of the shell, $\Delta$, is about a mean free path and within it incident particles are assumed to move in a free streaming manner unhindered by any frictional medium. The conventional diffusion equation is solved up to the point $r=a+b+\Delta$ and is linked to the condition inside the shell and up to the actual sphere surface by a current balance. Good results have been obtained with this technique for simple problems and there are strong reasons for expecting it to be valid for the more general problem discussed above. Reasonably accurate methods have been developed to solve the Brownian coagulation problem over the complete range of Knudsen numbers. These use the Fokker-Planck diffusion equation and differ in the method described here only in that the velocity of the particle explicitly appears as an independent variable. By means of such techniques it is possible to obtain a Brownian kernel that covers the hydrodynamic and Knudsen regimes in a single formula. Nevertheless, even here, it is necessary to make some additivity assumptions (Sitarski and Seinfeld 1977).

\section{Turbulent shear coagulation}

An important mechanism in aerosol coagulation occurs when the particles are entrained in turbulent eddies and are thrown around in the fluid in a random manner analogous to Brownian motion but according to the nature of the the turbulence. It is known (Levich 1962), for particles which are small compared with the turbulent microscale $\lambda_{0} \simeq\left(\nu^{3} / \varepsilon\right)^{1 / 4}$, where $\nu$ is the kinematic viscosity and $\varepsilon$ the turbulent energy dissipation rate per unit mass of gas, that the eddy diffusion coefficient can be deduced on the basis of isotropic turbulence and written as

$$
D_{\mathrm{T}}=k_{\mathrm{e}} r^{2}
$$

where $k_{\mathrm{e}}=\beta_{0}(\varepsilon / \nu)^{1 / 2}$ with $\beta_{0} \approx 0.15$. The symbol $r$ in equation (12) is the distance between the particle centres. For air at STP, $\nu=1.85 \times 10^{-5} \mathrm{~m}^{2} \mathrm{~s}^{-1}$ and we can obtain an estimate of $\varepsilon$ as follows. From dimensional arguments

$$
\varepsilon=\frac{\bar{u}^{3}}{\lambda_{\mathrm{T}}}
$$

where $\bar{u}$ is the root mean square turbulent velocity and $\lambda_{\mathrm{T}}$ is a length scale associated with the scale of production of energy containing eddies. If we assume a closed container of height $10 \mathrm{~m}$ and $\bar{u}=0.5 \mathrm{~m} \mathrm{~s}^{-1}$, then $\varepsilon \simeq 0.0125 \mathrm{~m}^{2} \mathrm{~s}^{-3}$. Thus the microscale of turbulence $\hat{\lambda}_{0} \simeq 840 \mu \mathrm{m}$. Since the particles under consideration are no greater than around $50 \mu \mathrm{m}$ and generally much smaller, we are clearly in the range of validity of equation (12).

Now if the particles are sufficiently small, they will also be undergoing classical Brownian diffusion and so the net diffusion coefficient will be the sum of the two effects, namely

$$
D(r)=D_{\mathrm{B}}+D_{\mathrm{T}}(r) .
$$

We note that whilst the Brownian diffusion coefficient, $D_{\mathrm{B}}$, depends on the sizes of the two particles, namely

$$
D_{\mathrm{B}}=D_{a}+D_{b}
$$

the turbulent diffusion coefficient is independent of $a$ and $b$.

Ignoring any other coagulation mechanisms we can now obtain the coagulation kernel using the formalism of $\S 2.1$. Because $D(r)$ depends only on the radial coordinate, the diffusion equation (equation (5)) reduces to

$$
\frac{1}{r^{2}} \frac{\mathrm{d}}{\mathrm{d} r}\left(r^{2} D(r) \frac{\mathrm{d} C}{\mathrm{~d} r}\right)=0
$$

Using the boundary conditions, this is readily integrated to give

$$
C(r)=C_{x}\left[1-\int_{r}^{\infty} \frac{\mathrm{d} w}{w^{2} D(w)}\left(\int_{a+b}^{\infty} \frac{\mathrm{d} w}{w^{2} D(w)}\right)^{-1}\right]
$$

from which

$$
K(a, b)=4 \pi\left(\int_{a+b}^{\infty} \frac{\mathrm{d} w}{w^{2} D(w)}\right)^{-1}
$$

In principle, the upper limit on the integral should be $\lambda_{0}$, and a further diffusion equation solved in the region $r>\lambda_{0}$ using a different form of $D_{\mathrm{T}}(t)$. However, it is readily shown that such corrections are of order $(a+b)^{3} / \lambda_{0}^{3}$ and hence negligible. 
Inserting equation (13) into equation (17) we find

$$
K(a, b)=4 \pi D_{\mathrm{B}}(a+b) g(\chi)
$$

where

$$
\chi=(a+b)\left(k_{\mathrm{e}} / D_{\mathrm{B}}\right)^{1 / 2}
$$

and

$$
g(\chi)=1 /\left(1-\pi \chi / 2+\chi \tan ^{-1} \chi\right)
$$

We readily note that, for small $\chi$, i.e. when the Brownian motion dominates the turbulent motion, the correction factor $g \rightarrow 1$. Thus we regain the classical Brownian coagulation kernel. On the other hand, for $\chi \rightarrow \infty$

$$
g(\chi) \rightarrow 3 \chi^{2}
$$

and hence

$$
\begin{gathered}
K(a, b) \simeq 12 \pi k_{\mathrm{e}}(a+b)^{3} \\
=5.65(a+b)^{3}\left(\frac{\varepsilon}{\nu}\right)^{1 / 2} .
\end{gathered}
$$

This is to be compared with a result obtained by Saffman and Turner (1956) namely

$$
\begin{gathered}
K(a, b) \simeq\left(\frac{8 \pi^{2}}{15}\right)^{1 / 2}(a+b)^{3}\left(\frac{\varepsilon}{\nu}\right)^{1 / 2} \\
=2.29(a+b)^{3}\left(\frac{\varepsilon}{\nu}\right)^{1 / 2} .
\end{gathered}
$$

Two comments can be made about this result. Firstly, it differs from equation (1) of Saffman and Turner who do not have $\pi^{2}$ in the radical but $\pi$. Following their arguments it would seem that there is a numerical slip present in their work. The second point is that our result differs from that of Saffman and Turner by a factor of 2.47. In view of the qualitative nature of the parameter $\beta_{0}$ in our work and the approximate statistical arguments in the work of Saffman and Turner, the agreement may be regarded as good. Certainly the functional dependence is reproduced and so we see that two rather different approaches lead to similar results. Moreover, by means of the diffusion equation, it has been possible to obtain a single expression for the coagulation kernel which spans Brownian and turbulent diffusion in terms of the parameter $\chi=(a+b)\left(k_{\mathrm{e}} / D_{\mathrm{B}}\right)^{1 / 2}$. The physical meaning of $\chi$ can be inferred from

$$
\chi^{2}=\frac{k_{\mathrm{e}}(a+b)^{2}}{D_{\mathrm{B}}}=\frac{D_{\mathrm{T}}^{*}}{D_{\mathrm{B}}}
$$

where $D_{\mathrm{T}}^{*}$ is the turbulent diffusion coefficient of touching spheres. Thus $\chi^{2}$ is the ratio of the local turbulent diffusion coefficient to the Brownian diffusion coefficient. To obtain an estimate of the error involved in the addition approximation normally used in solving equation (1), we use the ratio

$$
\gamma_{\mathrm{BT}}=\frac{K_{\mathrm{BT}}(a, b)}{K_{\mathrm{B}}(a, b)+K_{\mathrm{T}}(a, b)}
$$

where $K_{\mathrm{T}}(a, b)$ is the value for pure turbulent diffusion given by equation (20). In terms of the parameter $\chi$, we have

$$
\gamma_{\mathrm{BT}}=\frac{g(\chi)}{1+3 \chi^{2}} .
$$

Table 1 shows the results for a range of $\chi^{2}$ values. Clearly, the addition approximation underestimates the combined effect of Brownian and turbulent coagulation with the maximum error of $29 \%$ occurring at $\chi^{2} \simeq 0.2$.

Table 1. The error in the additivity assumption of Brownian and turbulent diffusion coagulation kernels.

\begin{tabular}{llllllll}
\hline$\chi^{2}$ & $\gamma_{B T}$ & $\chi^{2}$ & $\gamma_{B T}$ & $\chi^{2}$ & $\gamma_{B T}$ & $\chi^{2}$ & $\gamma_{B T}$ \\
\hline 0.001 & 1.048 & 0.01 & 1.138 & 0.1 & 1.282 & 1 & 1.165 \\
0.002 & 1.067 & 0.02 & 1.183 & 0.2 & 1.287 & 2 & 1.102 \\
0.003 & 1.081 & 0.03 & 1.211 & 0.3 & 1.271 & 3 & 1.074 \\
0.004 & 1.092 & 0.04 & 1.231 & 0.4 & 1.251 & 4 & 1.058 \\
0.005 & 1.102 & 0.05 & 1.246 & 0.5 & 1.233 & 5 & 1.048 \\
0.006 & 1.111 & 0.06 & 1.257 & 0.6 & 1.216 & 6 & 1.040 \\
0.007 & 1.119 & 0.07 & 1.266 & 0.7 & 1.201 & 7 & 1.035 \\
0.008 & 1.126 & 0.08 & 1.273 & 0.8 & 1.187 & 8 & 1.031 \\
0.009 & 1.132 & 0.09 & 1.278 & 0.9 & 1.175 & 9 & 1.028 \\
\hline
\end{tabular}

\section{Inclusion of the relative velocity}

Thus far, we have considered processes which involve pure diffusion. However, two important mechanisms exist in aerosol behaviour that involve a macroscopic relative velocity between the colliding particles. Thus gravitational forces lead to a relative velocity of

$$
\boldsymbol{V}=\boldsymbol{V}_{a}-\boldsymbol{V}_{b}
$$

which for gravity acting in the downward $z$-axis direction, denoted by the unit vector $k$, can be written

$$
\boldsymbol{V}=\frac{2 \rho_{\mathrm{p}} g}{9 \eta}\left|a^{2}-b^{2}\right| k \equiv\left|\tau_{a}-\tau_{b}\right| k
$$

where we have assumed Stokes law for the frictional drag. In the equation, $\rho_{\mathrm{p}}$ is the particle density and $g$ the acceleration due to gravity. It is assumed that the gas density is very much less than the particle density. The second mechanism leading to a relative velocity arises from turbulence and is due to the inertia of particles. This mechanism was first discussed quantitatively by East and Marshall (1954) and later by Saffman and Turner (1956); it recognises the fact that particles of different sizes will have different relative velocities in the turbulent eddies and hence collisions will be possible. Saffman and Turner (1956) show that the local particle velocity $\boldsymbol{q}_{a}$ can be related to the local acceleration of the gas $\mathrm{d} \boldsymbol{u} / \mathrm{d} t$, in the following way

$$
\boldsymbol{q}_{a}=\tau_{a} \frac{\mathrm{d} \boldsymbol{u}}{\mathrm{d} t}
$$


where

$$
\tau_{a}=\frac{2 \rho_{\mathrm{p}} a^{2}}{9 \eta}
$$

with $\rho_{\mathrm{p}}$ the particle density and ' $a$ ' its radius. Thus the mean square of the relative velocity between two particles ' $a$ ' and ' $b$ ' will be

$$
\overline{\left(\boldsymbol{q}_{a}-\boldsymbol{q}_{b}\right)^{2}}=\left(\tau_{a}-\tau_{b}\right)^{2} \overline{\left(\frac{\mathrm{d} \boldsymbol{u}}{\mathrm{d} t}\right)^{2}} .
$$

Using statistical arguments from the theory of homogeneous turbulence, Batchelor (1951) has shown that

$$
\overline{\left(\frac{\mathrm{d} u}{\mathrm{~d} t}\right)^{2}}=3.9 \frac{\varepsilon^{3 / 2}}{\nu^{1 / 2}} \text {. }
$$

Thus the relative velocity which promotes collisions can be taken as

$$
V_{\mathrm{T}}=1.97\left|\tau_{a}-\tau_{b}\right| \frac{\varepsilon^{3 / 4}}{\nu^{1 / 4}} .
$$

This is isotropically distributed and so will have no preferred direction. Saffman and Turner also dealt with gravitational effects in a similar statistical way. They regarded the acceleration due to gravity as an effective acceleration in the gas so that

$$
\overline{\left(\frac{\mathrm{d} u}{\mathrm{~d} t}\right)^{2}} \rightarrow \overline{\left(\frac{\mathrm{d} \boldsymbol{u}}{\mathrm{d} t}\right)^{2}}+g^{2}=3.9 \frac{\varepsilon^{3 / 2}}{\nu^{1 / 2}}+g^{2} .
$$

This approximation neglects the uni-directional effect of the gravitational force but ensures that, on average, the same number of encounters between particles arise. It is nevertheless an unfounded approximation and we shall examine it in more detail below. For the moment, we accept this approximation for gravity and write the net relative velocity, $V$, due to turbulent inertial motion and gravity as

$$
V=V_{\mathrm{T}}+V_{\mathrm{G}}=\left|\tau_{c}-\tau_{b}\right|\left(3.9 \frac{\varepsilon^{3 / 2}}{\nu^{1 / 2}}+g^{2}\right)^{1 / 2} .
$$

Finally, before using this result in the diffusion equation, we note that no account has been taken of the collision efficiency as discussed in $\S 2.3$. Such corrections, which we discuss below will lead to a relative velocity which depends on the separation distance of the particles. In order to proceed, it is necessary to return to equation (4), which we rewrite as

$$
\boldsymbol{\nabla} \cdot D(\boldsymbol{r}) \boldsymbol{\nabla} C(\boldsymbol{r})-\boldsymbol{V} \cdot \boldsymbol{\nabla} C-C \boldsymbol{\nabla} \cdot \boldsymbol{V}=0 .
$$

Now the relative velocity $V$ is independent of position and is assumed to be randomly distributed in direction, hence

$$
\boldsymbol{\nabla} \cdot \boldsymbol{V}=0 .
$$

Thus the equation for the concentration becomes

$$
\boldsymbol{\nabla} \cdot D(\boldsymbol{r}) D C(\boldsymbol{r})-\boldsymbol{V} \cdot \boldsymbol{\nabla} C(\boldsymbol{r})=0 .
$$

Since $D(r)$ is a function of inter-particle distance $r$, only, we can take coordinates such that

$$
\boldsymbol{V} \cdot \boldsymbol{\nabla C}=-V \frac{\partial C}{\partial r}
$$

whence equation (36) becomes

$$
\frac{1}{r^{2}} \frac{\mathrm{d}}{\mathrm{d} r}\left(r^{2} D(r) \frac{\mathrm{d} C(r)}{\mathrm{d} r}\right)+V \frac{\mathrm{d} C(r)}{\mathrm{d} r}=0 .
$$

The change in sign of $V$ occurs because, on average, the particle is moving towards the origin. There is a further modification required here which arises from the random nature of $V$. Saffman and Turner's assumption of randomness means that we can regard the problem as being analogous to the kinetic theory of gases where the number of particles crossing unit area per unit time in an isotropic distribution is $C V / 4$, where $V$ is the average velocity. The contribution to the current from drift, therefore, as defined by equation (3) should be $C V / 4$. Equation (37) differs in other respects from what might be expected. For example, in the case of electrical attraction between two particles where the force acts along the line of centres, the relative velocity $V=e_{r} V_{r}(r)$ and the equation for the concentration is, from equation (5), written as

$\frac{1}{r^{2}} \frac{\mathrm{d}}{\mathrm{d} r}\left(r^{2} D(r) \frac{\mathrm{d} C(r)}{\mathrm{d} r}\right)+\frac{1}{r^{2}} \frac{\mathrm{d}}{\mathrm{d} r}\left(r^{2} V_{r} C(r)\right)=0$.

The character of the solution of this equation differs from that of equation (37) because $\boldsymbol{\nabla} \cdot V \neq 0$.

If gravitational forces are treated properly, instead of in the approximate style of Saffman and Turner, it would be necessary to write

$$
\boldsymbol{\nabla} \cdot D(r) \boldsymbol{\nabla} C(r)+V_{\mathrm{G}} \boldsymbol{k} \cdot \boldsymbol{\nabla} C(r)=\cdot 0
$$

which in spherical polar coordinates $(r, \theta, \varphi)$ leads to

$$
\begin{aligned}
\frac{1}{r^{2}} \frac{\partial}{\partial r} & \left(r^{2} D(r) \frac{\partial C(r, \theta)}{\partial r}\right)+\frac{D(r)}{r^{2} \sin \theta} \frac{\partial}{\partial \theta} \\
& \times\left(\sin \theta \frac{\partial C(r, \theta)}{\partial \theta}\right)+V_{\mathrm{G}} \cos \theta \frac{\partial C(r, \theta)}{\partial r} \\
& -\frac{V_{\mathrm{G}} \sin \theta}{r} \frac{\partial C(r, \theta)}{\partial \theta}=0 .
\end{aligned}
$$

For $D(r)$ equal to a constant, we have solved this equation in a previous publication (Simons et al 1986) and will discuss our results below.

Returning to equation (37), we observe that it may be solved completely and that the coagulation kernel may be written

$$
\begin{aligned}
& \frac{1}{K(a, b)}=\frac{1}{4 \pi} \int_{a+b}^{x} \frac{\mathrm{d} w}{w^{2} D(w)} \\
& \quad \times \exp \left(-\frac{1}{4} \int_{a+b}^{w} \frac{V}{D\left(w^{1}\right)} \mathrm{d} w^{1}\right) .
\end{aligned}
$$

Using $D(w)=D_{\mathrm{B}}+k_{\mathrm{e}} w^{2}$ as before for Brownian and turbulent diffusion, we find

$$
K(a, b)=\frac{\pi(a+b)^{2} V}{G(\chi, \beta)}
$$


where

$$
\begin{array}{r}
G(\chi, \beta)=1-2 \exp \left(\frac{\beta}{4 \chi} \tan ^{-1} \chi\right) \\
\times \int_{1}^{\infty} \frac{\mathrm{d} t}{t^{3}} \exp \left(-\frac{\beta}{4 \chi} \tan ^{-1}(\chi t)\right)
\end{array}
$$

with $\beta=(a+b) V / D_{\mathrm{B}}$. Thus $\beta$ is a measure of the importance of inertial effects to Brownian diffusion effects and $\chi^{2}$ is a measure of the importance of turbulent diffusion effects to Brownian diffusion effects.

Equation (42) combines in one expression the effects of Brownian diffusion, turbulent diffusion, turbulent inertial and gravitational forces. The conventional method for dealing with these processes is to use a sum of the individual effects although Saffmann and Turner propose a root mean square sum.

For $\beta=\infty$, i.e. no Brownian diffusion, equation (42) reduces to

$$
K_{\mathrm{TG}}(a, b)=\frac{\pi V^{3}}{32 k_{\mathrm{e}}} \frac{1}{\left(1-\xi+\frac{1}{2} \xi^{2}-\mathrm{e}^{-\xi}\right)}
$$

where

$$
\xi=V / 4(a+b) k_{\mathrm{e}}=\beta / 4 \chi^{2} .
$$

For $\chi=0$, i.e. no turbulent diffusion, equation (42) reduces to

$$
K_{\mathrm{BG}}(a, b)=\frac{4 \pi(a+b) D_{\mathrm{B}} \mathrm{e}^{-\beta / 4}}{E_{2}(\beta / 4)}
$$

where $E_{2}(x)$ is an exponential integral function. For $\chi \rightarrow 0$ and $\beta \rightarrow 0$, equation (42) reduces to the classical result for Brownian diffusion

$$
K_{\mathrm{B}}(a, b)=4 \pi D_{\mathrm{B}}(a+b) \text {. }
$$

Similarly, for $\beta \rightarrow \infty$ and $\chi \rightarrow 0$, we regain the classical result

$$
K_{\mathrm{GI}}(a, b)=\pi(a+b)^{2} V .
$$

For gravitational forces only this yields the well known formula

$$
K_{\mathrm{G}}(a, b)=\pi(a+b)^{2} \frac{2 \rho_{\mathrm{p}} g}{9 \eta}\left|a^{2}-b^{2}\right|
$$

which is the exact result and to some extent must be fortuitous due to the statistical methods employed. For inertial turbulent coagulation, equation (47) together with equation (33) leads to

$$
K_{\mathrm{IT}}(a, b)=6.2(a+b)^{2}\left|\tau_{a}-\tau_{b}\right| \frac{\varepsilon^{3 / 4}}{\nu^{1 / 4}}
$$

which is to be compared with Saffman and Turner's result which has the constant 5.7 rather than 6.2 .

Finally, we can obtain the result for pure turbulent diffusion from equation (44) by allowing $\xi \rightarrow 0$, when we regain equation (20).

As a measure of the deviation of the sum kernel approximation from the result using the diffusion equation we compute

$$
\gamma(\xi, \beta)=\frac{K(a, b)}{K_{\text {sum }}(a, b)}
$$

where

$$
\begin{aligned}
& K_{\text {sum }}(a, b)=K_{\mathrm{B}}(a, b)+K_{\mathrm{T}}(a, b)+K_{\mathrm{G}}(a, b) \\
& =4 \pi D_{\mathrm{B}}(a+b)+12 \pi k_{\mathrm{e}}(a+b)^{3} \\
& \quad+\pi(a+b)^{2} V
\end{aligned}
$$

whence

$$
\gamma(\xi, \beta)=\frac{\beta}{\left(\beta+4+12 \chi^{2}\right) G(\chi, \beta)}
$$

where $\xi=\beta / 4 \chi^{2}$.

For turbulent diffusion and gravitational settling alone,

$$
\begin{gathered}
\gamma_{\mathrm{TG}}(\xi)=\frac{K_{\mathrm{TG}}(a, b)}{K_{\mathrm{T}}(a, b)+K_{\mathrm{G}}(a, b)} \\
=\frac{\xi^{3}}{2(3+\xi)\left(1-\xi+\frac{1}{2} \xi^{2}-\mathrm{e}^{-\xi}\right)}
\end{gathered}
$$

and for the Brownian diffusion and gravitational settling alone

$$
\gamma_{\mathrm{BG}}(\beta)=\frac{K_{\mathrm{BG}}(a, b)}{K_{\mathrm{B}}(a, b)+K_{\mathrm{G}}(a, b)}=\frac{\mathrm{e}^{-\beta / 4}}{(1+\beta / 4) E_{2}(\beta / 4)} .
$$

The form suggested by Saffmann and Turner's work was a root mean square average, namely

$$
K_{\mathrm{RMS}}(a, b)=\left(K_{\mathrm{T}}^{2}+K_{\mathrm{G}}^{2}\right)^{1 / 2}
$$

whence

$$
\gamma_{\mathrm{RMS}}(\xi)=\frac{\xi^{3}}{2\left(9+\xi^{2}\right)^{1 / 2}} \frac{1}{\left(1-\xi+\frac{1}{2} \xi^{2}-\mathrm{e}^{-\xi}\right)}
$$

which is to be compared with equation (53).

\subsection{Numerical illustrations}

In order to gain some insight into the accuracy of the above procedure, it is useful to evaluate $\gamma_{\mathrm{BG}}(\beta)$ first and compare the results with the exact values (Simons et al 1986). Table 2 shows the results. We see that, overall, the agreement is very good. For $\beta<2$, equation (54) overestimates the true value by $5.3 \%$ at worst; for $\beta>3$ the error changes sign increasing to a maximum of $7.7 \%$ at $\beta \simeq 30$ and thereafter reducing. The simple and accurate nature of equation (54), compared with the slowly converging infinite sum which represents the exact value, makes its use highly desirable; we shall also later discuss a method for including kinetic theory corrections when the Knudsen number is large.

To compare the combined effects of turbulent diffusion, turbulent inertial and gravitational effects, we show table 3 which illustrates equations (53) and (56) 
Table 2. A comparison of the Brownian and gravitational kernel obtained by the approximate method of equation (45) with the exact results of Simons et al (1986).

\begin{tabular}{rlll}
\hline$\beta$ & $\gamma_{\text {exact }}$ & $\gamma_{\text {approx }}$ & $\Delta \% \dagger$ \\
\hline 0.1 & 1.0236 & 1.0609 & -3.6 \\
0.2 & 1.0447 & 1.0943 & -4.7 \\
0.3 & 1.0637 & 1.1190 & -5.2 \\
0.4 & 1.0808 & 1.1384 & -5.3 \\
0.5 & 1.0963 & 1.1543 & -5.3 \\
0.6 & 1.1110 & 1.1675 & -5.1 \\
0.7 & 1.1230 & 1.1787 & -5.0 \\
0.8 & 1.1347 & 1.1882 & -4.7 \\
0.9 & 1.1453 & 1.1964 & -4.5 \\
1 & 1.1551 & 1.2034 & -4.2 \\
1.5 & 1.1933 & 1.2267 & -2.9 \\
2 & 1.2189 & 1.2379 & -1.6 \\
3 & 1.2487 & 1.2433 & 0.5 \\
4 & 1.2628 & 1.2387 & 1.9 \\
5 & 1.2687 & 1.2304 & 3.1 \\
6 & 1.2702 & 1.2209 & 3.9 \\
7 & 1.2691 & 1.2113 & 4.6 \\
8 & 1.2666 & 1.2019 & 5.1 \\
9 & 1.2631 & 1.1930 & 5.5 \\
10 & 1.2591 & 1.1846 & 5.9 \\
20 & 1.2182 & 1.1270 & 7.5 \\
50 & 1.1507 & 1.0649 & 7.5 \\
100 & 1.1072 & 1.0358 & 6.4 \\
\hline
\end{tabular}

Table 3. The error in the additivity and root mean square approximations for turbulent diffusion and gravitational coagulation kernels.

\begin{tabular}{rll}
\hline$\xi$ & $\gamma_{\text {TG }}$ & $\gamma_{\text {RMS }}$ \\
\hline 0.1 & 0.992 & 1.025 \\
0.2 & 0.985 & 1.048 \\
0.3 & 0.978 & 1.071 \\
0.4 & 0.972 & 1.092 \\
0.5 & 0.967 & 1.113 \\
0.6 & 0.962 & 1.132 \\
0.7 & 0.957 & 1.150 \\
0.8 & 0.953 & 1.167 \\
0.9 & 0.950 & 1.182 \\
1 & 0.946 & 1.197 \\
2 & 0.925 & 1.283 \\
3 & 0.918 & 1.299 \\
4 & 0.918 & 1.285 \\
5 & 0.920 & 1.262 \\
6 & 0.923 & 1.239 \\
7 & 0.927 & 1.217 \\
8 & 0.931 & 1.199 \\
9 & 0.935 & 1.182 \\
10 & 0.938 & 1.168 \\
20 & 0.961 & 1.093 \\
50 & 0.982 & 1.039 \\
70 & 0.987 & 1.028 \\
100 & 0.990 & 1.020 \\
\hline
\end{tabular}

numerically. We note that the sum kernel overestimates the coagulation by up to $8 \%$ at about $\xi=4$. On the other hand, the Saffman and Turner root mean square approximation underestimates the coagulation by up to $30 \%$ at $\xi=3$.

The complete kernel, including all coagulation mechanisms, is represented by equation (52). We illustrate this result in table 4 , using the non-dimensional parameters $\beta$ and $\xi$ rather than $\beta$ and $\chi$. It is clear that nowhere in the $\beta-\xi$ plane is the error in the sum kernel greater than $29 \%$ and that the sum kernel everywhere underestimates the true kernel.

\section{Extension of the method}

There are two important aspect of the problem that have been omitted in the above discussion. This was deliberate since the purpose of this paper is to illustrate the method using simple examples. Nevertheless, we can comment on the deficiencies. In this respect, we have neglected kinetic theory effects and particle-fluid interactions. These problems have been generally discussed in $\S \S 2.3$ and 2.4. As far as the modifications to equation (5) are concerned, we can include to a good first approximation the effect of kinetic theory by modifying the boundary conditions given by equation (7). This is written instead as

$$
C(\boldsymbol{r})=\lambda \boldsymbol{n} \cdot \boldsymbol{\nabla C}(\boldsymbol{r})
$$

where $\lambda$ is a 'jump' distance which depends weakly on the radii of the two particles but is generally of the order of a mean free path. An expression for $\lambda$ can be obtained via the Fuch's technique discussed above. It is not difficult to modify the solutions obtained above to obey condition (57). In addition to this modified boundary condition, it is also necessary to recognise that the mobility, $B$, of the particles, i.e. the velocity per unit force, is a function of the Knudsen number. Since both the diffusion coefficient and the viscous drag force depend on the mobility, namely

$$
\begin{gathered}
D=K T B \\
F_{\mathrm{DRAG}}=V / B
\end{gathered}
$$

it is necessary to obtain $B$ from a separate calculation. Cercignani et al (1968) have performed such a calculation for a sphere and obtained the drag force over the complete range of Knudsen numbers. Indeed, they also show that the original Milliken result, i.e.

$$
B=\frac{1}{6 \pi a \eta}\left(1+\frac{l_{0}}{a}\left(A+Q \mathrm{e}^{-b a / l_{0}}\right)\right)
$$

is in excellent agreement with the exact theory. Here $l_{0}$ is the mean free path. Thus there seems no insuperable difficulty in dealing adequately with the coagulation of very fine particles.

A more difficult problem concerns the collision efficiency, for this requires knowledge of the interparticle fluid forces which can also influence the mobility 
Table 4. The error $\gamma(\xi, \beta)$ in the additivity approximation for Brownian diffusion, turbulent diffusion, turbulent inertial and gravitational coagulation kernels.

\begin{tabular}{|c|c|c|c|c|c|c|c|c|}
\hline & 0.1 & 0.2 & 0.5 & 1 & 2 & 5 & 10 & 20 \\
\hline 0.001 & 1.075 & 1.054 & 1.035 & 1.025 & 1.018 & 1.012 & 1.009 & 1.006 \\
\hline 0.002 & 1.103 & 1.075 & 1.049 & 1.035 & 1.025 & 1.017 & 1.012 & 1.009 \\
\hline 0.005 & 1.152 & 1.114 & 1.076 & 1.055 & 1.040 & 1.027 & 1.020 & 1.016 \\
\hline 0.01 & 1.199 & 1.153 & 1.104 & 1.077 & 1.057 & 1.039 & 1.029 & 1.023 \\
\hline 0.02 & 1.246 & 1.199 & 1.141 & 1.106 & 1.080 & 1.055 & 1.043 & 1.034 \\
\hline 0.05 & 1.286 & 1.259 & 1.201 & 1.157 & 1.121 & 1.087 & 1.069 & 1.058 \\
\hline 0.1 & 1.276 & 1.284 & 1.246 & 1.203 & 1.162 & 1.121 & 1.100 & 1.084 \\
\hline 0.2 & 1.226 & 1.271 & 1.275 & 1.246 & 1.208 & 1.162 & 1.137 & 1.120 \\
\hline 0.5 & 1.135 & 1.197 & 1.259 & 1.270 & 1.254 & 1.219 & 1.195 & 1.178 \\
\hline 1 & 1.078 & 1.127 & 1.202 & 1.242 & 1.255 & 1.244 & 1.231 & 1.219 \\
\hline 2 & 1.039 & 1.070 & 1.129 & 1.177 & 1.213 & 1.235 & 1.239 & 1.239 \\
\hline 3 & 1.025 & 1.045 & 1.090 & 1.133 & 1.173 & 1.210 & 1.225 & 1.233 \\
\hline 4 & 1.017 & 1.032 & 1.066 & 1.102 & 1.141 & 1.184 & 1.206 & 1.221 \\
\hline 5 & 1.012 & 1.023 & 1.049 & 1.080 & 1.116 & 1.162 & 1.188 & 1.207 \\
\hline 6 & 1.009 & 1.017 & 1.038 & 1.063 & 1.096 & 1.142 & 1.172 & 1.193 \\
\hline 7 & 1.007 & 1.013 & 1.029 & 1.050 & 1.080 & 1.126 & 1.157 & 1.180 \\
\hline 8 & 1.005 & 1.010 & 1.022 & 1.040 & 1.066 & 1.111 & 1.143 & 1.168 \\
\hline 9 & 1.003 & 1.007 & 1.017 & 1.031 & 1.055 & 1.098 & 1.132 & 1.157 \\
\hline 10 & 1.002 & 1.005 & 1.012 & 1.024 & 1.045 & 1.087 & 1.121 & 1.147 \\
\hline 20 & 1.001 & 1.003 & 1.007 & 1.018 & 1.030 & 1.023 & 1.055 & 1.084 \\
\hline
\end{tabular}

$B$. The effect of such forces leads to the Brownian diffusion coefficient and the relative velocity in equation (4) becoming complicated functions of position. The calculation of $D(r)$ for Brownian coagulation has been carried out by Spielman (1970) and by Shahub and Williams (1988) and, given the specific details of the inter-particle forces due to London-van der Waals interaction, reliable values for this term are available. On the other hand, the relative velocity under gravitational forces involves solutions of the equations of motion for two particles coupled by the parallel and perpendicular forces between two spheres. This can be done, but it is a very complex calculation which it is not appropriate to discuss here.

However, it is possible to use a simple expression for the relative velocity of two particles given by Batchelor (1976). There it is shown that the radial velocities $U_{a r}$ and $U_{b r}$ of spheres of radii $a$ and $b$, whose centres are a distance $r$ apart, are given by

$$
U_{a r}=\frac{A_{a a}(p, r)}{6 \pi a \eta} F_{a r}+\frac{A_{a b}(p, r)}{3 \pi \eta(a+b)} F_{b r}
$$

and

$$
U_{b r}=\frac{A_{b b}(p, r)}{6 \pi b \eta} F_{b r}+\frac{A_{b a}(p, r)}{6 \pi \eta(a+b)} F_{a r}
$$

where $p=b / a$ and the $A_{j i}(p, r)$ are known from previous work on spheres moving along their line of centres (Stimson and Jeffrey 1926, Cooley and O'Neill 1969). Using Saffman and Turner's approach we set $F_{a r}=$ $m_{a b} g$ and $F_{b r}=m_{b} g$ for gravitational effects and to the appropriate values for turbulent inertial collisions. Thus we can write for the relative velocity

$$
\begin{gathered}
U_{a r}-U_{b r}=\left(A_{a a}-\frac{2 a A_{b a}}{a+b}\right) \frac{m_{a} g}{6 \pi a \eta} \\
-\left(A_{b b}-\frac{2 b A_{a b}}{a+b}\right) \frac{m_{b} g}{6 \pi b \eta} .
\end{gathered}
$$

Of course this expression ignores the inertial effect arising from acceleration terms and is limited in that respect. The effective diffusion coefficient has also been obtained by Batchelor and can be written

$$
D(r)=D_{\mathrm{B}}\left(\frac{p A_{a a}+A_{b b}}{1+p}-\frac{4 p A_{a b}}{(1+p)^{2}}\right) .
$$

Now whilst detailed expressions are available for the $A_{i j}$ they are extremely complicated and it is useful for illustrative purposes to use the simpler, asymptotic form, namely

$$
\begin{gathered}
A_{a a}=1-\frac{15 p^{3}}{4(1+p)^{4}} \frac{(a+b)^{4}}{r^{4}} \\
A_{b a}=\frac{3 a+b}{4}-\frac{\left(1+p^{2}\right)}{4(1+p)^{2}} \frac{(a+b)^{3}}{r^{3}} .
\end{gathered}
$$

$A_{b b}$ and $A_{a b}$ are obtained from (65) and (66) by interchanging $a$ and $b$. From these expressions we find

$$
\left|U_{a r}-U_{b r}\right|=V_{\propto} f\left(\frac{a+b}{r}\right)
$$

where

$$
f\left(\frac{1}{x}\right)=1-\frac{3 x}{2(1+p)}+\frac{1+p^{2}}{2(1+p)^{3}} x^{3}
$$


and $V_{x}$ is given by

$$
V_{\infty}=\frac{2 \rho_{\mathrm{p}} g}{9 \eta}\left|a^{2}-b^{2}\right|
$$

but we can also replace it by the $V$ of equation (33) to include turbulent inertia. Similarly

$$
D(r)=D_{\mathrm{B}} g\left(\frac{a+b}{r}\right)
$$

where

$$
\begin{aligned}
g\left(\frac{1}{x}\right) & =1-\frac{3 p x}{(1+p)^{2}}+\frac{p\left(1+p^{2}\right)}{(1+p)^{4}} x^{3} \\
& -\frac{15 p\left(1+p^{3}\right)}{4(1+p)^{5}} x^{5}
\end{aligned}
$$

As an estimate of the accuracy of these expressions, we show in table 5 the values of $g(1 / x)$ versus $x$ and also versus $h$, the gap distance between the sphere surfaces divided by $a$, for the case $a=b$. It is clear that the approximation is very good for $h>0.25$. Using equation (41) and the values of diffusion coefficient and relative velocity given by equations (67) and (68), we find (assuming $\boldsymbol{\nabla} \cdot \boldsymbol{V} \simeq 0$ ) that the coagulation kernel $K(a, b)$ takes the form

$$
\frac{K_{\mathrm{B}}(a, b)}{K(a, b, p)}=\int_{0}^{1} \frac{\mathrm{d} t}{g(1 / t)} \exp \left(-\frac{\beta}{4} \int_{t}^{1} \frac{\mathrm{d} w}{w^{2}} \frac{f(1 / w)}{g(1 / w)}\right)
$$

A collision efficiency can be defined as the ratio of the coagulation kernel with fluid effects to that without. Thus

$$
\begin{aligned}
& \varepsilon(\beta, p)=\mathrm{e}^{\beta / 4} E_{2}(\beta / 4) \\
& \times\left[\int_{0}^{1} \frac{\mathrm{d} t}{g(1 / t)} \exp \left(-\frac{\beta}{4} \int_{1}^{1} \frac{\mathrm{d} w}{w^{2}} \frac{f(1 / w)}{g(1 / w)}\right)\right]^{-1} .
\end{aligned}
$$

For $\beta p^{3} \gg 1$, i.e. negligible Brownian motion, this expression reduces to

$$
\varepsilon(p)=1+\frac{1+p^{2}}{2(1+p)^{3}}-\frac{3}{2(1+p)}
$$

which varies from zero to $\frac{3}{8}$ as $p$ varies from zero to unity. This compares favourably with other simple approximations such as that of Pruppacher and Klett (1978) who obtain

$$
\varepsilon_{\mathrm{PK}}=\frac{p^{2}}{2\left(1+p^{2}\right)}
$$

by less than rigorous means. Note, however, that equation (74) is not strictly correct for $p \rightarrow 0$ because of the restriction $\beta p^{3} \gg 1$. We shall comment on this again below.

One important observation regarding the form of $\varepsilon(p)$ is that it depends only on $p=b / a$ whereas it is known from more accurate calculations and physical reasoning that it should also depend on the absolute value of the particle size. Our results do not show this effect because we have neglected the inertial terms in obtaining the relative velocity. An improved calculation of this velocity would remove this defect. Table 6 shows values of $\varepsilon(\beta, p)$ for a range of $\beta$ and $p$. It is clear that even for quite large values of $\beta$ (i.e. inertia dominates diffusion) the diffusive effect increases the collision efficiency above that for pure gravitational coagulation for small $p$.

As a measure of the error involved in using the sum kernel for Brownian and gravitational coagulation, we compute

$$
\begin{aligned}
\gamma_{\mathrm{BG}}(\beta, p) & =\frac{K(a, b ; p)}{K_{\mathrm{B}}(a, b)+\varepsilon(p) K_{\mathrm{G}}(a, b)} \\
& =\frac{1}{I(\beta, p)(1+\varepsilon \beta / 4)}
\end{aligned}
$$

where $I(\beta, p)$ is equal to the integral in equation (73).

Table 7 shows the results and indicates that the sum kernel, even with the gravitational term corrected by a collision efficiency, can be significantly in error. For $\beta \leqslant 20$, there are errors of more than $100 \%$ depending on the value of $p$. Moreover, we see that, even for large values of $\beta$, the error becomes very large for small values of $p$. This is related to the condition $\beta p^{3} \gg 1$ derived earlier and physically it means that, even for large $\beta$ there is significant Brownian diffusion of the smaller particles which is not accounted for by the sum kernel and which therefore leads that approximation to underestimate the coagulation rate. We also calculate the collision efficiency with a constant value of the relative velocity but a position-dependent diffusion coefficient. This removes the $\boldsymbol{\nabla} \cdot \boldsymbol{V} \approx 0$ approximation and enables us to examine the influence of the variable diffusion coefficient throughout the whole range of $\beta$ values. Moreover, the case $\beta=0$ corresponds to purely Brownian motion and so is of considerable interest. Table 8 shows values of the collision efficiency for a 
Table 6. The collision efficiency $\varepsilon(\beta, p)$ for combined Brownian diffusion and gravitational settling with spatially variable relative velocity and diffusion coefficient.

\begin{tabular}{|c|c|c|c|c|c|c|c|c|c|c|}
\hline & 1 & 2 & 5 & 10 & 20 & 50 & 100 & 200 & 300 & $x$ \\
\hline 0.01 & 0.820 & 0.732 & 0.581 & 0.454 & 0.337 & 0.211 & 0.144 & 0.0972 & 0.0773 & $1.95 \times 10^{-4}$ \\
\hline 0.02 & 0.803 & 0.717 & 0.569 & 0.446 & 0.330 & 0.207 & 0.141 & 0.0957 & 0.0762 & $7.62 \times 10^{-4}$ \\
\hline 0.05 & 0.756 & 0.674 & 0.535 & 0.420 & 0.311 & 0.197 & 0.135 & 0.0926 & 0.0743 & $4.43 \times 10^{-3}$ \\
\hline 0.1 & 0.688 & 0.613 & 0.488 & 0.385 & 0.288 & 0.185 & 0.130 & 0.0917 & 0.0753 & 0.0158 \\
\hline 0.2 & 0.586 & 0.524 & 0.422 & 0.338 & 0.260 & 0.177 & 0.133 & 0.103 & 0.0900 & 0.0509 \\
\hline 0.3 & 0.519 & 0.467 & 0.383 & 0.314 & 0.251 & 0.185 & 0.150 & 0.127 & 0.118 & 0.0942 \\
\hline 0.4 & 0.476 & 0.432 & 0.361 & 0.305 & 0.254 & 0.202 & 0.176 & 0.160 & 0.154 & 0.140 \\
\hline 0.5 & 0.449 & 0.412 & 0.353 & 0.307 & 0.266 & 0.226 & 0.208 & 0.197 & 0.193 & 0.185 \\
\hline 0.6 & 0.434 & 0.401 & 0.352 & 0.314 & 0.282 & 0.253 & 0.241 & 0.235 & 0.233 & 0.229 \\
\hline 0.7 & 0.426 & 0.397 & 0.356 & 0.325 & 0.301 & 0.282 & 0.275 & 0.272 & 0.271 & 0.269 \\
\hline 0.8 & 0.423 & 0.398 & 0.363 & 0.339 & 0.322 & 0.310 & 0.307 & 0.307 & 0.307 & 0.307 \\
\hline 0.9 & 0.423 & 0.401 & 0.372 & 0.353 & 0.342 & 0.337 & 0.338 & 0.340 & 0.340 & 0.342 \\
\hline 1 & 0.426 & 0.407 & 0.382 & 0.368 & 0.362 & 0.363 & 0.367 & 0.372 & 0.372 & 0.375 \\
\hline
\end{tabular}

Table 7. The ratio of exact Brownian and gravitational kernel to the sum kernel.

\begin{tabular}{llllllllll}
\hline$p$ & 1 & 2 & 5 & 10 & 20 & 50 & 100 & 200 & 300 \\
\hline 0.01 & 1.233 & 1.359 & 1.608 & 1.886 & 2.277 & 3.026 & 3.859 & 5.002 & 5.864 \\
0.02 & 1.208 & 1.331 & 1.574 & 1.846 & 2.223 & 2.948 & 3.726 & 4.790 & 5.549 \\
0.05 & 1.136 & 1.249 & 1.473 & 1.722 & 2.057 & 2.684 & 3.273 & 3.940 & 4.294 \\
0.1 & 1.031 & 1.129 & 1.325 & 1.536 & 1.805 & 2.221 & 2.511 & 2.664 & 2.655 \\
0.2 & 0.870 & 0.949 & 1.098 & 1.243 & 1.401 & 1.555 & 1.576 & 1.509 & 1.437 \\
0.3 & 0.763 & 0.828 & 0.949 & 1.054 & 1.154 & 1.221 & 1.204 & 1.156 & 1.126 \\
0.4 & 0.692 & 0.750 & 0.851 & 0.937 & 1.010 & 1.056 & 1.054 & 1.040 & 1.031 \\
0.5 & 0.646 & 0.700 & 0.794 & 0.870 & 0.934 & 0.980 & 0.995 & 0.998 & 0.998 \\
0.6 & 0.618 & 0.668 & 0.758 & 0.829 & 0.890 & 0.943 & 0.967 & 0.983 & 0.989 \\
0.7 & 0.600 & 0.650 & 0.737 & 0.805 & 0.867 & 0.929 & 0.958 & 0.977 & 0.984 \\
0.8 & 0.591 & 0.641 & 0.726 & 0.795 & 0.858 & 0.921 & 0.952 & 0.975 & 0.983 \\
0.9 & 0.586 & 0.636 & 0.721 & 0.789 & 0.853 & 0.917 & 0.952 & 0.975 & 0.981 \\
1 & 0.586 & 0.636 & 0.720 & 0.788 & 0.851 & 0.918 & 0.953 & 0.973 & 0.983 \\
\hline
\end{tabular}

Table 8. The collision efficiency for constant relative velocity and spatially variable diffusion coefficient.

\begin{tabular}{|c|c|c|c|c|c|c|c|c|}
\hline$\beta$ & 0 & 1 & 2 & 5 & 10 & 20 & 50 & 100 \\
\hline 0.01 & 0.980 & 0.980 & 0.981 & 0.983 & 0.986 & 0.990 & 0.994 & 0.996 \\
\hline 0.02 & 0.961 & 0.961 & 0.962 & 0.967 & 0.973 & 0.980 & 0.988 & 0.993 \\
\hline 0.05 & 0.908 & 0.907 & 0.911 & 0.923 & 0.937 & 0.953 & 0.973 & 0.984 \\
\hline 0.1 & 0.831 & 0.830 & 0.838 & 0.860 & 0.886 & 0.916 & 0.953 & 0.972 \\
\hline 0.2 & 0.712 & 0.714 & 0.729 & 0.768 & 0.812 & 0.864 & 0.926 & 0.957 \\
\hline 0.3 & 0.630 & 0.635 & 0.655 & 0.707 & 0.766 & 0.833 & 0.910 & 0.949 \\
\hline 0.4 & 0.574 & 0.583 & 0.607 & 0.668 & 0.737 & 0.815 & 0.902 & 0.945 \\
\hline 0.5 & 0.538 & 0.549 & 0.576 & 0.644 & 0.720 & 0.804 & 0.897 & 0.942 \\
\hline 0.6 & 0.514 & 0.527 & 0.556 & 0.629 & 0.709 & 0.797 & 0.894 & 0.941 \\
\hline 0.7 & 0.499 & 0.514 & 0.544 & 0.619 & 0.703 & 0.794 & 0.892 & 0.940 \\
\hline 0.8 & 0.490 & 0.506 & 0.537 & 0.614 & 0.699 & 0.792 & 0.891 & 0.940 \\
\hline 0.9 & 0.486 & 0.502 & 0.534 & 0.612 & 0.697 & 0.791 & 0.891 & 0.939 \\
\hline 1 & 0.484 & 0.501 & 0.533 & 0.611 & 0.697 & 0.790 & 0.891 & 0.939 \\
\hline
\end{tabular}


range of $\beta$ - and $p$-values. We note that, for $\beta=0$, the collision efficiency is close to unity for small values of $p$ but as $p$ tends to unity the collision efficiency tends to 0.484 . This indicates that Brownian diffusion is much less effective in promoting coagulation than the classical theory would suggest. For $\beta>0$, the results show by comparison with table 6 that a variable diffusion coefficient without the corresponding variable relative velocity (and vice versa) can lead to significant errors in the collision efficiency. This is due to the fact that the ranges of influence of $D(r)$ and $V(r)$ are similar and hence they tend to interfere, either enhancing or diminising their individual effects.

\section{Summary and conclusions}

The mechanisms which promote aerosol coagulation; namely, Brownian diffusion, gravitational sedimentation and turbulence, act on the particles simultaneously and cannot, therefore, be treated independently. In this paper, we have considered these mechanisms and devised a formalism which enables a unified coagulation kernel to be derived. This avoids the ad hoc procedure normally employed of taking the arithmetic sum of the coagulation kernels derived in isolation.

The method employs a diffusion equation with due allowance for mass flow by a directed velocity. In this way, we can account for diffusive processes due to Brownian motion and turbulent diffusion as well as effects which depend upon the inertia of the particle, namely gravitational settling and turbulence. Turbulent inertia is regarded as a statistical effect with the flux of particles calculated in a manner which is analogous to calculation of molecular flux. Less satisfactory is our treatment of gravitational sedimentation, because in order to obtain an analytic solution of the diffusion equation we have had to treat that in a statistical manner following the work of Saffman and Turner. However, comparison of the result with an exact solution for a special case indicates that the error is small.

We have further indicated how the effects of interparticle fluid forces influence the results and how they may be included in the formalism. Such forces make the diffusions coefficient and the relative velocity functions of inter-particle distance but the formalism is general enough to encompass this fact. Preliminary results with simple models of the inter-particle fluid forces indicate that significant differences exist between these results and those of the classical methods. In particular. there is a strong interaction between Brownian motion and gravitational settling in the transition region and, as expected, the collision efficiency is significantly less than unity.

\section{References}

Batchelor G K 1951 Proc. Camb. Phil. Soc. 47359 - 1976 J. Fluid Mech. 74

Cercignani C 1975 Theory and Applications of the Boltzmann Equation (Edinburgh: Scottish Academic)

Cercignani C, Pagani C D and Bassanini P 1968 Phys. Fluids 111399

Chandrasekhar C 1943 Rev. Mod. Phys. 151

Cooley M D A and O'Neil M E 1969 Proc. Camb. Phil. Soc. 66407

Crump J G and Seinfeld J H $1981 \mathrm{~J}$. Aerosol Sci. 12405

East T W R and Marshall J S 1954 Q. J. R. Meteorol. Soc. 8026

Friedlander S K 1977 Smoke, Dust and Haze (New York: Wiley)

Hidy G M and Brock J R 1970 The Dynamics of Aerocolloidal Systems vol 1 (New York: Pergamon)

Hocking L M 1959 Q. J. R. Meteorol. Soc. 8544

Levich V G 1962 Physiochemical Hydrodynamics (Englewood Cliffs, NJ: Prentice-Hall)

Lushnikov A 1974 J. Colloid Sci. 48400

Papoulis A 1965 Probability, Random Variables and Stochastic Processes (New York: McGraw-Hill)

Pertmer G A and Loyalka S K 1980 Nucl. Technol. 4770

Pruppacher H R and Klett J D 1978 Microphysics of Clouds and Precipitation (Dordrecht: Reidel)

Saffman P G and Turner J S 1956 J. Fluid Mech. 116 Shahub A and Williams M M R $1988 J$. Phys. D: Appl. Phys. 21231

Silberberg M (ed.) 1979 Nuclear Aerosols in Reactor Safety, $O E C D$ Report of the European Nuclear Energy Agency

Simons S. Williams M M R and Cassell J S 1986 J. Aerosol Sci. 17789

Sitarski M and Seinfeld J H 1977 J. Colloid Interface Sci. 61261

Spielman L A 1970 J. Colloid Interface Sci. 33562

Stimson M and Jeffrey G B 1926 Proc. R. Soc. A 111110 